\title{
Evidence for nonhadronic degrees of freedom in the transverse mass spectra of kaons from relativistic nucleus-nucleus collisions?
}

\author{
E. L. Bratkovskaya, S. Soff, and H. Stöcker \\ Institut für Theoretische Physik, Universität Frankfurt, 60054 Frankfurt, Germany \\ M. van Leeuwen \\ NIKHEF, Amsterdam, Netherlands \\ W. Cassing \\ Institut für Theoretische Physik, Universität Giessen, 35392 Giessen, Germany
}

\begin{abstract}
We investigate transverse hadron spectra from relativistic nucleus-nucleus collisions which reflect important aspects of the dynamics - such as the generation of pressure - in the hot and dense zone formed in the early phase of the reaction. Our analysis is performed within two independent transport approaches (HSD and UrQMD) that are based on quark, diquark, string and hadronic degrees of freedom. Both transport models show their reliability for elementary $p p$ as well as light-ion $(\mathrm{C}+\mathrm{C}, \mathrm{Si}+\mathrm{Si})$ reactions. However, for central $\mathrm{Au}+\mathrm{Au}(\mathrm{Pb}+\mathrm{Pb})$ collisions at bombarding energies above $\sim 5 \mathrm{~A} \cdot \mathrm{GeV}$ the measured $K^{ \pm}$transverse mass spectra have a larger inverse slope parameter than expected from the calculation. Thus the pressure generated by hadronic interactions in the transport models above $\sim 5 \mathrm{~A} \cdot \mathrm{GeV}$ is lower than observed in the experimental data. This finding shows that the additional pressure - as expected from lattice QCD calculations at finite quark chemical potential and temperature - is generated by strong partonic interactions in the early phase of central $\mathrm{Au}+\mathrm{Au}(\mathrm{Pb}+\mathrm{Pb})$ collisions.

PACS numbers: 25.75.-q, 25.75.Dw, 25.75.Ld, 13.60.Le
\end{abstract}

The phase transition from partonic degrees of freedom (quarks and gluons) to interacting hadrons is a central topic of modern high-energy physics. In order to understand the dynamics and relevant scales of this transition laboratory experiments under controlled conditions are presently performed with ultra-relativistic nucleusnucleus collisions. Hadronic spectra and relative hadron abundancies from these experiments reflect important aspects of the dynamics in the hot and dense zone formed in the early phase of the reaction. Furthermore, as has been proposed early by Rafelski and Müller [1] the strangeness degree of freedom might play an important role in distinguishing hadronic and partonic dynamics.

Estimates based on the Bjorken formula 2] for the energy density achieved in central $\mathrm{Au}+\mathrm{Au}$ collisions suggest that the critical energy density for the formation of a quark-gluon plasma (QGP) is by far exceeded during a few $\mathrm{fm} / \mathrm{c}$ in the initial phase of the collision at Relativistic Heavy Ion Collider (RHIC) energies 3], but sufficient energy densities $\left(\sim 0.7-1 \mathrm{GeV} / \mathrm{fm}^{3}[4]\right)$ might already be achieved at Alternating Gradient Synchrotron (AGS) energies of $\sim 10 A \cdot \mathrm{GeV}[5,6]$. More recently, lattice QCD calculations at finite temperature and quark chemical potential $\mu_{q}$ [7] show a rapid increase of the thermodynamic pressure $P$ with temperature above the critical temperature $T_{c}$ for a phase transition to the QGP. The crucial question is, however, at what bombarding energies the conditions for the phase transition are fulfilled.

Presently, transverse mass (or momentum) spectra of hadrons are in the center of interest: the measured transverse mass $m_{T}$ spectra of kaons

$$
\frac{1}{m_{T}} \frac{d N}{d m_{T}} \sim \exp \left(-\frac{m_{T}}{T}\right)
$$

at AGS and SPS (from 30 to $160 A \cdot \mathrm{GeV}$ at the Super Proton Synchrotron) energies show a substantial flattening or hardening of the spectra in central $\mathrm{Au}+\mathrm{Au}$ collisions relative to $p p$ interactions (cf. [8, 9]). This hardening of the spectra (or increase of the inverse slope parameter $T$ in (1) is commonly attributed to strong collective flow which is absent in the respective $p p$ data. We will demonstrate in this Letter that the pressure needed to generate such a large collective flow cannot be produced by the interactions of hadrons in the expansion phase of the hadronic fireball.

Two independent transport models that employ hadronic and string degrees of freedom, i.e. UrQMD [10, 11] and HSD [12, 13], are used for our study. They take into account the formation and multiple rescattering of hadrons and thus dynamically describe the generation of pressure in the hadronic expansion phase. The UrQMD transport approach [10, 11] includes all baryonic resonances up to masses of $2 \mathrm{GeV}$ as well as mesonic resonances up to $1.9 \mathrm{GeV}$ as tabulated by the Particle Data Group [14]. For hadronic continuum excitations a string model is used with hadron formation times in the order of 1-2 fm/c depending on the momentum and energy of the created hadron. The transport approach is matched to reproduce the nucleon-nucleon, mesonnucleon and meson-meson cross section data in a wide kinematic range [10, 11]. In the HSD approach nucleons, $\Delta$ 's, $\mathrm{N}^{*}(1440), \mathrm{N}^{*}(1535), \Lambda, \Sigma$ and $\Sigma^{*}$ hyperons, $\Xi$ 's, $\Xi^{*}$ 's and $\Omega$ 's as well as their antiparticles are in- 

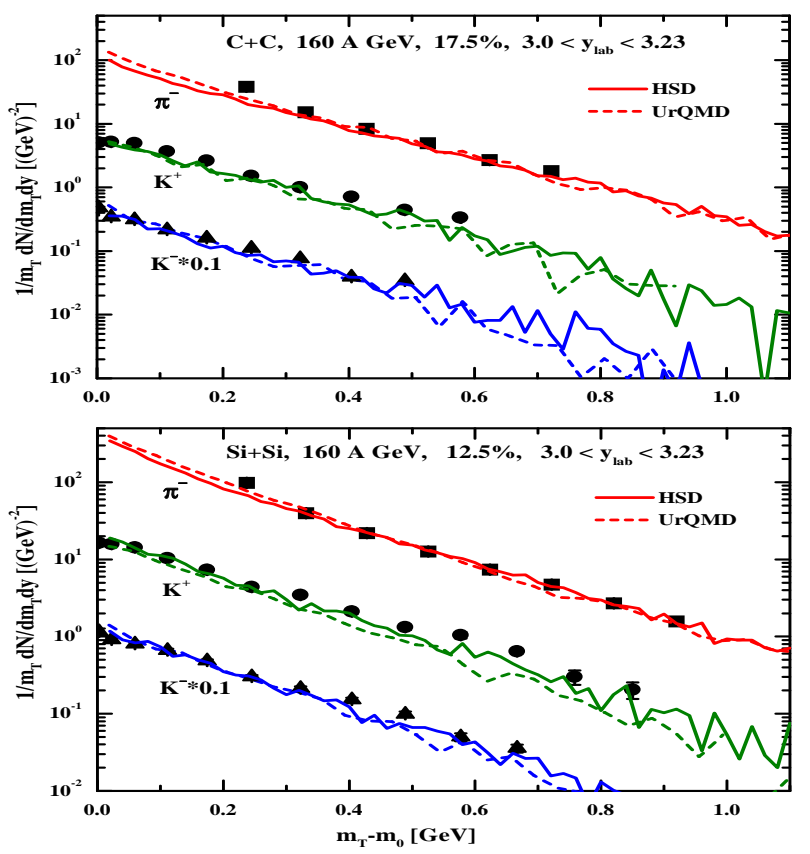

FIG. 1: Comparison of transverse mass spectra from HSD (solid lines) and UrQMD (dashed lines) for $\pi^{-}, K^{+}$and $K^{-}(\times 0.1)$ from $17.5 \%$ central $\mathrm{C}+\mathrm{C}$ (upper part) and $12.5 \%$ central Si+Si (lower part) reactions at $160 A \cdot \mathrm{GeV}$ close to midrapidity with data from Ref. [19] (full symbols).

cluded on the baryonic side whereas the $0^{-}$and $1^{-}$octet states are included in the mesonic sector. High energy inelastic hadron-hadron collisions in HSD are described by the FRITIOF string model [15] whereas low energy hadron-hadron collisions are modeled based on experimental cross sections. We point out importantly, that no parton-parton scattering processes are included in the studies below contrary to the multi-phase transport model (AMPT) 16], which is currently employed from upper SPS to RHIC energies.

Whereas the underlying concepts of the two transport theoretical models are very similar, the actual realizations differ considerably. Indeed, a systematic analysis of results from both models and experimental data for central nucleus-nucleus collisions from 2 to $160 A \cdot \mathrm{GeV}$ has shown that the 'longitudinal' rapidity distributions of protons, pions, kaons, antikaons and hyperons are quite similar in both models and in reasonable agreement with available data. The exception are the pion rapidity spectra at the highest AGS energy and lower SPS energies, which are overestimated by both models 17]. For a comparison of HSD calculations with experimental data at RHIC energies we refer the reader to Ref. [18].

We focus on transverse mass spectra of pions and kaons/antikaons from central $\mathrm{Au}+\mathrm{Au}(\mathrm{Pb}+\mathrm{Pb})$ collisions from $2 A \cdot \mathrm{GeV}$ to $21.3 A \cdot \mathrm{TeV}$ and compare to recent data. We start with results on $m_{T}$ spectra for central $\mathrm{C}+\mathrm{C}$ and $\mathrm{Si}+\mathrm{Si}$ collisions at $160 A \cdot \mathrm{GeV}$. In Fig. 1 the experimental $m_{T}$ spectra for $\pi^{-}, K^{+}$and $K^{-}$for $\mathrm{C}+\mathrm{C}$ reactions (17.5\% centrality; upper part) and $\mathrm{Si}+\mathrm{Si}(12.5 \%$ central-

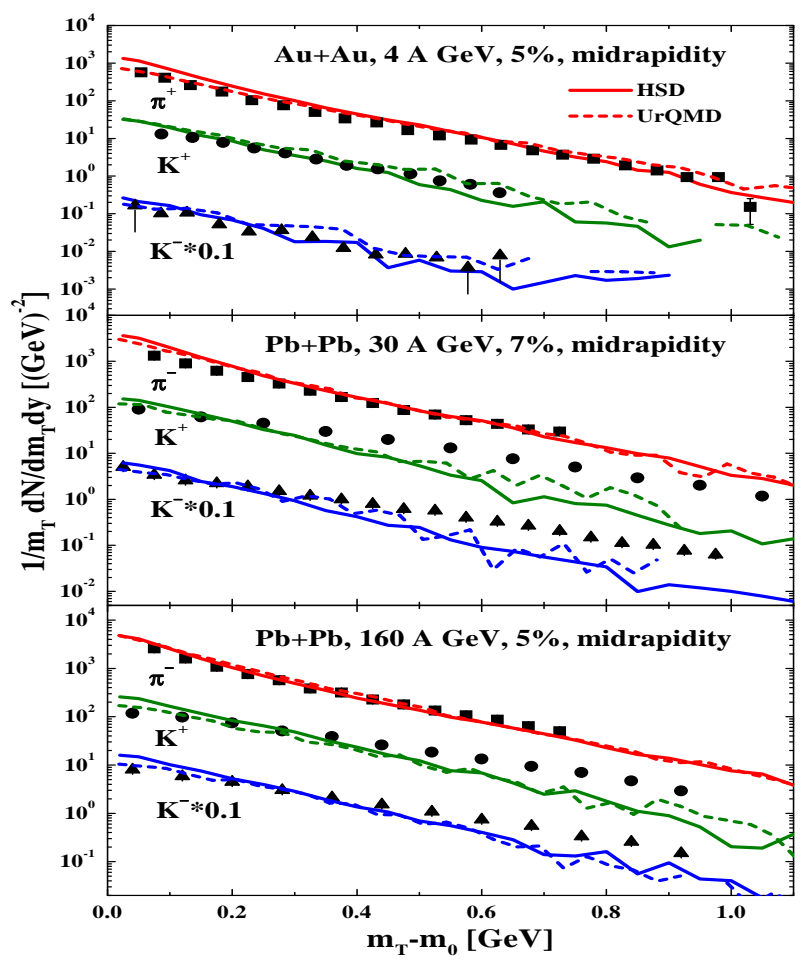

FIG. 2: Comparison of transverse mass spectra from HSD (solid lines) and UrQMD (dashed lines) for $\pi^{ \pm}, K^{+}$and $K^{-}(\times 0.1)$ from central $\mathrm{Au}+\mathrm{Au}(\mathrm{Pb}+\mathrm{Pb})$ reactions at 4, 30, and $160 A \cdot \mathrm{GeV}$ at midrapidity with the data from Refs. 8, 20] (full symbols).

ity; lower part) [19] are compared to HSD (solid lines) and UrQMD calculations (dashed lines). The $m_{T}$ spectra are calculated in a rapidity interval $\left(3.0 \leq y_{\text {lab }} \leq\right.$ $3.23)$ close to midrapidity. The agreement between the transport calculations and the data is quite satisfactory; no obvious traces of 'new' physics are visible. The situation, however, changes for central $\mathrm{Au}+\mathrm{Au}$ (or $\mathrm{Pb}+\mathrm{Pb}$ ) collisions as demonstrated in Fig. 2 where we display the calculated spectra from UrQMD (dashed lines) and HSD (solid lines) for 5\% (or $7 \%$ ) central $\mathrm{Au}+\mathrm{Au}$ (or $\mathrm{Pb}+\mathrm{Pb}$ ) collisions at 4,30 and $160 A \cdot \mathrm{GeV}$ in comparison to midrapidity data from Refs. [8, 20] 32]. Whereas at the lowest energy of $4 \mathrm{~A} \cdot \mathrm{GeV}$ the agreement between the transport approaches and the data is still acceptable, severe deviations are visible in the $K^{ \pm}$spectra at SPS energies of 30 and $160 A \cdot G e V$. Note that the $\pi^{ \pm}$spectra are reasonably described at all energies while the inverse slope $T$ of the $K^{ \pm}$transverse mass spectra is underestimated severely by about the same amount in both transport approaches (within statistics). The increase of the inverse $K^{ \pm}$slopes in heavy-ion collisions with respect to $p p$ collisions, which is generated by rescatterings of produced hadrons in the transport models, is only small because the elastic meson-baryon scattering is strongly forward peaked and therefore gives little additional transverse momentum at midrapidity.

The question remains whether the discrepancies shown in Fig. 2 might be due to conventional hadronic medium 
effects. In fact, the $m_{T}$ slopes of kaons and antikaons at SIS energies ( 1.5 to $2 A \cdot \mathrm{GeV})$ were found to differ significantly 21]. As argued in 13] the different slopes could be traced back to repulsive potentials of kaons with nucleons, which lead to a hardening of the $K^{+}$spectra, and attractive antikaon-nucleon potentials, which lead to a softening of the $K^{-}$spectra. However, the effect of such potentials was calculated within HSD and was found to be of minor importance at AGS and SPS energies 13. since the meson densities are comparable to or even larger than the baryon densities at AGS energies and above.

Additional self energy contributions stem from $K^{ \pm}$interactions with mesons; however, $s$-wave kaon-pion interactions are weak due to chiral symmetry arguments and $p$-wave interactions such as $\pi+K \leftrightarrow K^{*}$ transitions are suppressed substantially by the approximately 'thermal' pion spectrum. A recent study on the kaon potentials in hot pion matter gives kaon mass shifts of about $-52 \mathrm{MeV}$ and vector potentials of $\sim+49 \mathrm{MeV}$ [22] for a pion gas at temperature $T=170 \mathrm{MeV}$. We have employed even slightly larger $K^{ \pm}$potentials in dynamical HSD calculations and achieved a hardening of the $K^{ \pm}$spectra of less than $10 \%$.

Furthermore, we have pursued the idea of Refs. [23, 24] that the $K^{ \pm}$spectra could be hardened by string-string interactions, which increase the effective string tension $\sigma$ and thus the probability to produce mesons at high $m_{T}$ [16, 24]. In order to estimate the largest possible effect of string-string interactions we have assumed that for two overlapping strings the string tension $\sigma$ is increased by a factor of two, for three overlapping strings by a factor of three etc. Here the overlap of strings is defined geometrically assuming a transverse string radius $R_{s}$, which according to the studies in Ref. [25] should be $R_{s} \leq 0.25$ fm. Based on these assumptions (and $R_{s}=0.25 \mathrm{fm}$ ), we find only a small increase of the inverse slope parameters at AGS energies, where the string densities are low. At $160 A \cdot \mathrm{GeV}$, the model gives a significant hardening of the spectra by about $15 \%$, which, however, is still much less than in the data in Fig. 2

Our findings are summarized in Fig. 3] where the dependence of the inverse slope parameter $T$ (see Eq. (1)) on $\sqrt{s}$ is shown and compared to experimental data 8, 19] for central $\mathrm{Au}+\mathrm{Au}(\mathrm{Pb}+\mathrm{Pb})$ collisions (l.h.s.) and $p p$ reactions (r.h.s.). The upper and lower solid lines (with open circles) on the l.h.s. in Fig. 3 correspond to results from HSD calculations, where the upper and lower limits are due to fitting the slope $T$ itself, an uncertainty in the repulsive $K^{ \pm}$-pion potential or the possible effect of string overlaps. The open triangles represent slope parameters from default UrQMD calculations (version 1.3) which are well within the limits obtained from the different HSD calculations. It's worth to point out that in the pure cascade mode the kaon slope $T$ is slightly larger in the UrQMD calculations than from HSD at AGS and SPS energies, which is related to the isotropic decay of higher mass $(<2 \mathrm{GeV})$ resonances in UrQMD as well as a different string fragmentation function. The slope
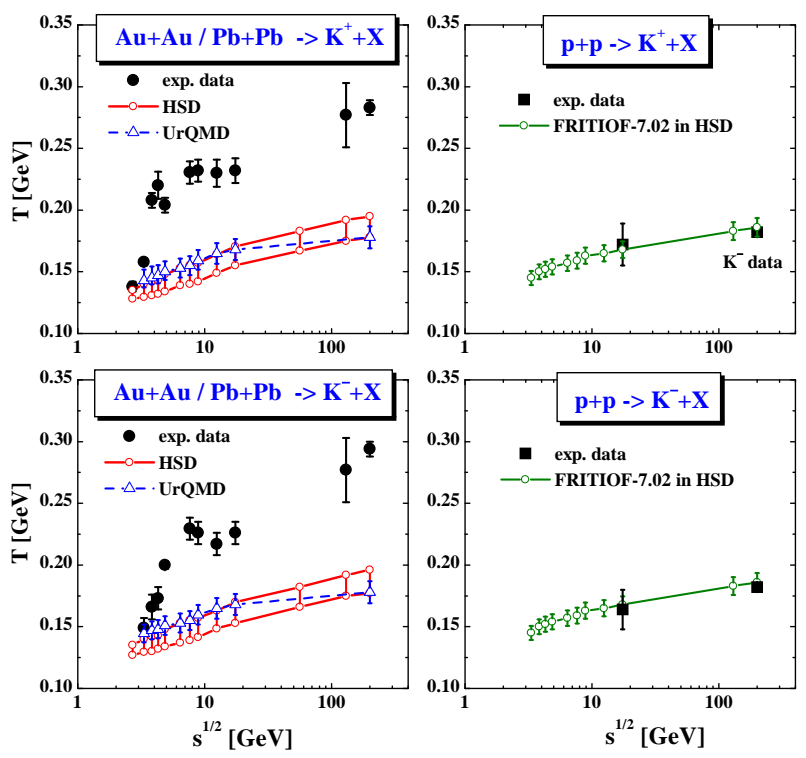

FIG. 3: Comparison of the inverse slope parameters for $K^{+}$ and $K^{-}$mesons from central $\mathrm{Au}+\mathrm{Au}(\mathrm{Pb}+\mathrm{Pb})$ collisions (l.h.s.) and $p p$ reactions (r.h.s.) as a function of the invariant energy $\sqrt{s}$ from HSD (upper and lower solid lines) and UrQMD (open triangles) with data from Refs. [8, 19]. The upper and lower solid lines result from different limits of the HSD calculations as discussed in the text.

parameters from $p p$ collisions (r.h.s. in Fig. 3) are seen to increase smoothly with energy both in the experiment (full squares) and in the HSD calculations (full lines with open circles). The error bars related to our calculations are due to an uncertainty in extracting the slope parameter when considering different $m_{T}$ intervals.

We mention that the RQMD model [23] gives higher inverse slope parameters for kaons at AGS and SPS energies than HSD and UrQMD, which essentially can be traced back to the implementation of resonances with masses above $2 \mathrm{GeV}$ as well as 'color ropes' that decay isotropically in their rest frame 26]. However, we consider the implementation of such 'heavy resonances' with unknown properties as a phenomenological fit to experimental data.

This still leaves us with the question of the origin of the rapid increase of the $K^{ \pm}$slopes with invariant energy for central $\mathrm{Au}+\mathrm{Au}$ collisions, which is missed in both transport approaches. We recall that higher transverse particle momenta either arise from repulsive self energies - in mean-field dynamics - or from collisions, which reduce longitudinal momenta in favor of transverse momenta. As shown in Fig. 3 conventional hadron selfenergy effects and hadronic binary collisions are insufficient to describe the dramatic increase of the $K^{ \pm}$slopes as a function of $\sqrt{s}$. We conclude that this points towards a different mechanism for the generation of the pressure observed experimentally and propose, that partonic degrees of freedom should be responsible for this effect already at $\sim 5 \mathrm{~A} \cdot \mathrm{GeV}$.

Our arguments are based on a comparison of the trans- 
verse pressure from the transport models with recent lattice QCD calculations 77: The transverse pressure $\left(P_{x x} \approx P_{y y}\right)$ - in the central overlap volume of $\mathrm{Au}+\mathrm{Au}$ collisions - within UrQMD is about $0.07 \mathrm{GeV} / \mathrm{fm}^{3}$ at 10.7 A GeV, $0.1 \mathrm{GeV} / \mathrm{fm}^{3}$ at $40 \mathrm{~A} \mathrm{GeV}$ and $0.15 \mathrm{GeV} / \mathrm{fm}^{3}$ at $160 \mathrm{~A} \mathrm{GeV}$ (cf. Ref. 27]). These numbers should be compared to lattice QCD calculations. Adopting the results from Ref. 7] and using $T_{c} \approx 172 \mathrm{MeV}$ (following Ref. 28]) we end up with a pressure (in thermal and chemical equilibrium) of $0.15 \mathrm{GeV} / \mathrm{fm}^{3}$ at vanishing baryon density $\left(\mu_{B}=0\right), 0.17 \mathrm{GeV} / \mathrm{fm}^{3}\left(\mu_{B}=0.21\right.$ $\mathrm{GeV}), 0.2 \mathrm{GeV} / \mathrm{fm}^{3}\left(\mu_{B}=0.33 \mathrm{GeV}\right), 0.22 \mathrm{GeV} / \mathrm{fm}^{3}$ $\left(\mu_{B}=0.42 \mathrm{GeV}\right)$, and $0.26 \mathrm{GeV} / \mathrm{fm}^{3}\left(\mu_{B}=0.53 \mathrm{GeV}\right)$ at a temperature $T=1.1 T_{c}(\sim 190 \mathrm{MeV})$. These values are higher than those from the transport calculations, but correspond to a temperature significantly above $T_{c}$. The important finding from the lattice QCD calculations [7] is that the pressure drops by an order of magnitude when the temperature decreases to $T_{c}$, which leads to $\sim 0.02-0.03 \mathrm{GeV} / \mathrm{fm}^{3}$ at $T_{c}$. These numbers now are lower than the transport results for bombarding energies $\geq 10.7 A \cdot \mathrm{GeV}$ ! Consequently, the phase transition should be reached even at a lower bombarding energy in line with our findings in Fig. 3.

The authors of Ref. 29] have proposed to interpret the approximately constant $K^{ \pm}$slopes above $\sim 30 A \cdot \mathrm{GeV}$ as an indication for a phase transition 29]. However, to support their arguments more experimental data in the energy range $20 \mathrm{GeV} \leq \sqrt{s} \leq 100 \mathrm{GeV}$ are needed to clarify this issue experimentally.

Summarizing this study, we conclude that according to two independent string-hadron transport models, HSD and UrQMD, the inverse slope parameters $T$ for $K^{ \pm}$ mesons are practically independent of system size from $p p$ up to central $\mathrm{Pb}+\mathrm{Pb}$ collisions and show a slight increase with collision energy. The calculated transverse mass spectra are in reasonable agreement with the experimental results for $p p$ collisions and central collisions of light nuclei $(\mathrm{C}+\mathrm{C}$ and $\mathrm{Si}+\mathrm{Si})$. The rapid increase of the inverse slope parameters of kaons for collisions of heavy nuclei $(\mathrm{Au}+\mathrm{Au})$ found experimentally in the AGS energy range, however, is not reproduced by both models (see Fig. 31). Since the pion transverse mass spectra are described sufficiently well at all bombarding energies, the failure has to be attributed to a lack of pressure especially for the strange $K^{ \pm}$-mesons. We have argued - based on lattice QCD calculations for the pressure at finite temperature and baryon chemical potential $\mu_{B}$ from Ref. [7] - that this additional pressure must be generated in the early phase of the collision because the strong hadronic interactions in the later stages don't produce it.

The interesting finding of our analysis is, that nonhadronic degrees of freedom seem to play a substantial role in central $\mathrm{Au}+\mathrm{Au}$ collisions already at AGS energies above $\sim 5 A \cdot \mathrm{GeV}$. Further indications for a low energy onset of a phase transition are the missed $K^{+} / \pi^{+}$ratios in the AGS and lower SPS regime [17. Additionally, the excitation function of the elliptic flow $v_{2}$ indicates a softening of the equation of state at $\sim 5 A \cdot \mathrm{GeV}$ in $\mathrm{Au}+\mathrm{Au}$ collisions [30, 31], which signals a change of the pressure, too. In spite of the latter observations - all showing a significant change at $\sim 5 \mathrm{~A} \cdot \mathrm{GeV} \mathrm{Au}+\mathrm{Au}$ collisions - data for weakly interacting (on the hadronic level) $\phi$-mesons will be necessary to clarify further the question raised in the title from the experimental side.

The authors like to thank M. Bleicher, C. Greiner, C.M. Ko, Z.W. Lin and H. Weber for valuable discussions. E.L.B. was supported by DFG.
[1] J. Rafelski and B. Müller, Phys. Rev. Lett. 48, 1066 (1982).

[2] J.D. Bjorken, Phys. Rev. D 27, 140 (1983).

[3] Quark Matter 2002, Nucl. Phys. A715, 1 (2003).

[4] F. Karsch et al., Nucl. Phys. B 502, 321 (2001).

[5] H. Stöcker and W. Greiner, Phys. Rept. 137, 277 (1986).

[6] W. Cassing et al., Nucl. Phys. A 674, 249 (2000).

[7] Z. Fodor, S. D. Katz, and K. K. Szabo, Phys. Lett. B 568, 73 (2003).

[8] V. Friese et al., NA49 Collaboration, nucl-ex/0305017

[9] M. I. Gorenstein et al., Phys. Lett. B 567, 175 (2003).

[10] S.A. Bass et al., Prog. Part. Nucl. Phys. 42, 255 (1998).

[11] M. Bleicher et al., J. Phys. G 25, 1859 (1999).

[12] J. Geiss et al., Nucl. Phys. A 644, 107 (1998).

[13] W. Cassing and E. L. Bratkovskaya, Phys. Rep. 308, 65 (1999).

[14] K. Hagiwara et al., (Review of Particle Properties), Phys. Rev. D 66, 010001 (2002).

[15] B. Andersson et al., Z. Phys. C 57, 485 (1993).

[16] Z. W. Lin et al., Nucl. Phys. A 698, 375 (2002).

[17] H. Weber et al., Phys. Rev. C 67, 014904 (2003).

[18] E. L. Bratkovskaya et al., Phys. Rev. C 67, 054905
(2003).

[19] I. Kraus et al., NA49 Collaboration, nucl-ex/0306022

[20] L. Ahle et al. E866 and E917 Collaboration, Phys. Lett. B 476, 1 (2000); ibid. 490, 53 (2000).

[21] A. Förster et al., KaoS Collaboration, J. Phys. G 28, 2011 (2002).

[22] B. V. Martemyanov et al., nucl-th/0212064

[23] H. Sorge, Phys. Rev. C 52, 3291 (1995).

[24] S. Soff et al., Phys. Lett B 471, 89 (1999).

[25] J. Geiss et al., Phys. Lett. B447, 31 (1999).

[26] H. van Hecke et al., Phys. Rev. Lett. 81, 5764 (1998).

[27] L. V. Bravina et al., Phys. Rev. C 60, 024904 (1999).

[28] J. Latessier and J. Rafelski, Phys. Rev. C 67, 031902(R) (2003).

[29] M. Gazdzicki and M. I. Gorenstein, Acta Phys. Polon. B 30, 2705 (1999).

[30] P. Danielewicz et al., Phys. Rev. Lett 81, 2438 (1998).

[31] P. K. Sahu and W. Cassing, Nucl. Phys. A 712, 357 (2002).

[32] Note that all data from the NA49 Collaboration at 30 $\mathrm{A} \cdot \mathrm{GeV}$ have to be considered as 'preliminary'. 